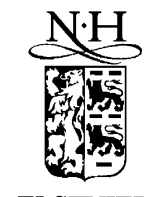

\title{
Ultra-deep LIGA process and its applications
}

\author{
Y. Cheng ${ }^{\mathrm{a}, \mathrm{b}, *}$, B.-Y. Shew ${ }^{\mathrm{a}}$, M.K. Chyu ${ }^{\mathrm{c}}$, P.H. Chen ${ }^{\mathrm{d}}$ \\ ${ }^{\mathrm{a}}$ Microstructure Group, Synchrotron Radiation Research Center, \#1, R\&D Rd. VI, Science-Based Industrial Park, \\ Hsinchu 300, Taiwan, ROC \\ ${ }^{\mathrm{b}}$ Department of Engineering and System Science, National Tsing Hua University, Hsinchu 300, Taiwan, ROC \\ ${ }^{\mathrm{c}}$ Department of Mechanical Engineering, University of Pittsburgh, PA 15261, USA \\ ${ }^{\mathrm{d}}$ Department of Mechanical Engineering, National Taiwan University, Taipei 106, Taiwan, ROC
}

\begin{abstract}
Deep X-ray lithography developed in Germany around the 1980s is an important micromachining technology. Combined with electroplating and molding, this technology can produce micromachining parts with low cost and flexible choices of material. The ultra-deep (UD) LIGA process developed at the Synchrotron Radiation Research Center (SRRC) that provides better development of photoresist is capable of producing microstructures of millimeters in size. The ongoing micromachining projects at SRRC benefited from this UD LIGA process include: microfiber spinneret, leadframe punch, and w-band klystrino. (C) 2001 Elsevier Science B.V. All rights reserved.
\end{abstract}

Keywords: Ultra-deep X-ray lithography; Thick-film lithography; Ultra-deep LIGA process; Synchrotron radiation; Micromachining

\section{UD LIGA process}

While microtechnology research at the Synchrotron Radiation Research Center (SRRC) was initiated in 1996 [1], a complete on-site cleanroom facility will not be available until the year 2001 . Presently our needs in microlithography use the nearby facilities at Tsing-Hua University. Mainly due to resource limitation, our research focus at SRRC has been directed primarily to extending the structural depth, rather than to improving feature precision, say down to the submicron level.

\footnotetext{
*Corresponding author. Synchrotron Radiation Research Centre, Microstructure Group, No. 1, R\&D Road VI, Hsinchu Science Based Ind. Park, Hsinchu, 30077, Taiwan, ROC. Tel.: +886-35-780-281; fax: +886-35-789-816.

E-mail address: yao@srrc.gov.tw (Y. Cheng).
}

This strategy also falls in line with the local industrial needs in Taiwan. In terms of scaling of micro devices, Taiwanese industry is interested mainly in two types of microstructures: ten micron-deep and millimeter-deep microstructures. Since the typical single exposure depth of Taiwan Light Source (characteristic wavelength of $0.5 \mathrm{~nm}$ ) without filters is about $200 \mu \mathrm{m}$, an alternative for achieving these depths is to use successive exposures in conjunction with the conformal mask technology. Our experience reveals that there are two major technical barriers for making millimeter-deep structures: (1) wet developing process, and (2) thermal stresses. As a result of extensive research over the past years, the wet development problem can be alleviated by increasing the exposure dosage and reducing the diffractioninduced dosage absorption on the wall [2]. To 
establish the X-ray mask fabrication capability in house, a low-cost gold mask of 30 microns using laser-write has been developed. This approach is known as the "ultra-deep LIGA process (UD LIGA)" [3]. Overall this method is superior in precision to the contact printing, due mainly to the tracking of lithography plan. However, cracks may be developed from severe thermal stresses as the structure depth approaches three millimeters.

Our focus of modeling thick-film lithography is based largely on analytical formulations of physical phenomena, rather than computer simulation. This approach with direct physical modeling is capable of revealing explicit guidelines for quality control, technology extension, and user training. Specifically, we have developed a universal dosage formula for SR X-ray exposure [4]. This formula is equally useful for different synchrotron facilities and materials. Furthermore, we have developed a theoretical estimation of the wall-profile based on Fresnel diffraction and exposure attenuation [5]. Again, the same formula is also applicable to other lithographic processes including those based on deep X-ray and UV. Information on precision, resolution and process optimization can be easily obtained using this formula.

\subsection{Development}

The time of development is by no means a linear function of resist thickness. This is especially the case for developing a high-aspect-ratio microtrench. The developing rate is limited by two major factors, i.e. attenuation of the irradiation dosage and mass transfer in the trench. As an example, a thee-millimeter microtrench irradiated at SRRC was not realized even after a nearly oneweek developing time. Eventually the resist degraded seriously in the developing bath and became practically useless. To reduce the dosage attenuation, it requires irradiation by a hard X-ray source. However, on the other hand, the adverse effects of the high-energy photoelectrons are undesirable [2]. With all the factors considered, a successive exposure scheme has been chosen to boost the dosage for increasing the developing speed, particularly deep in the trench. This led to a reduction of developing time to one-third of the original duration.

\subsection{Thermal stresses}

There is a large discrepancy between the thermal expansion coefficients of resist and substrate. To keep the substrate flat under various ambient temperatures, a sandwich structure to balance the thermal stresses from both sides of the substrate was developed [3]. Such an approach can uniformly reduce the magnitudes of thermal stresses in the resist. As mentioned, our experience revealed that cracks might easily occur due to uncompensated stresses, as the resist is over three millimeters thick.

\section{Microfiber spinneret}

The world production as well as demand of synthetic fibers grows continuously. For instance, annual growth rate of polyester fibers production has been more than $5 \%$ in recent years. Taiwan is one of the largest polyester manufacturers in the world, which supplies $21 \%$ and $14 \%$ of global demands of polyester filaments and staple, respectively. It is expected that the polyester production will increase from 2 to 3 million tons in the next two years. In addition, Taiwan's polyester producers are the most competitive worldwide because of their continuous investment in technology. With such high fiber productivity, a special applause is justified, as Taiwan is unable to produce the spinneret which is one of the key components for producing fibers. In the new millennium, the competition from Mainland China and Korea is growing, due mainly to lower labor costs. Therefore, Taiwan has to focus on developing those highly profitable fibers with variable cross sections. One of the key technological advantages of micromachined spinneret is its capability of producing microfibers and fibers with functionality.

The micromachining laboratory at SRRC has been developing the LIGA technology with special focuses on the domestic market needs [6]. The most notable features of the deep X-ray lithography are producing high depths and high aspect 
ratios microstructures. While there are many applications that can be benefited by UD LIGA, the spinneret is one of the demands.

To date, spinnerets for spinning of fine fibers are mainly manufactured by drill, wire-cut, and EDM. Although recent developments in micro EDM in Taiwan has increased the aspect ratio and the lateral precision, the productivity as well as the uniformity of micro EDM remains relatively limited. Therefore, mass production of spinnerets often requires additional molding process post EDM. Additional assembly steps for the final products may follow this. Recently LIGA technology has been developed for batch production of spinnerets. Compared to the aforementioned, conventional methods, LIGA has many advantages which include high uniformity, capability of fabricating arbitrary cross sections, low surface roughness and low cost. However, the popularity of LIGA technology may be hampered by the limited accessibility of synchrotron light source. This situation may be alleviated in the future as the technology of tabletop light source becomes available.

Another important advantage that LIGA can offer is concerned with product's aspect ratio. To prevent undesirable blockage in a direct spinning process, the capillary width of a microfiber spinneret generally is greater than $100 \mu \mathrm{m}$. Therefore, making the capillary deeper is much more important than making the capillary smaller. From the theoretical standpoint, the aspect ratio of a shaped capillary needs to be larger than 6 ensuring that the spinning flow in the capillary is predominantly fully-developed. The conventional methods of drilling or EDM can only produce capillary with an aspect ratio of about 3 while LIGA technology can result in a much higher aspect ratio nearly 20 .

Figs. 1 and 2 combined show the design of a spinneret capillary with a complex shaped cross section. A complex cross section is often needed to attain special functionality of the fiber. Spinnerets for either direct spinning or composite spinning can now be manufactured routinely using UD LIGA. Fig. 3 illustrates the high throughput LIGA-based production method. In terms of production speed and quality, LIGA outperforms

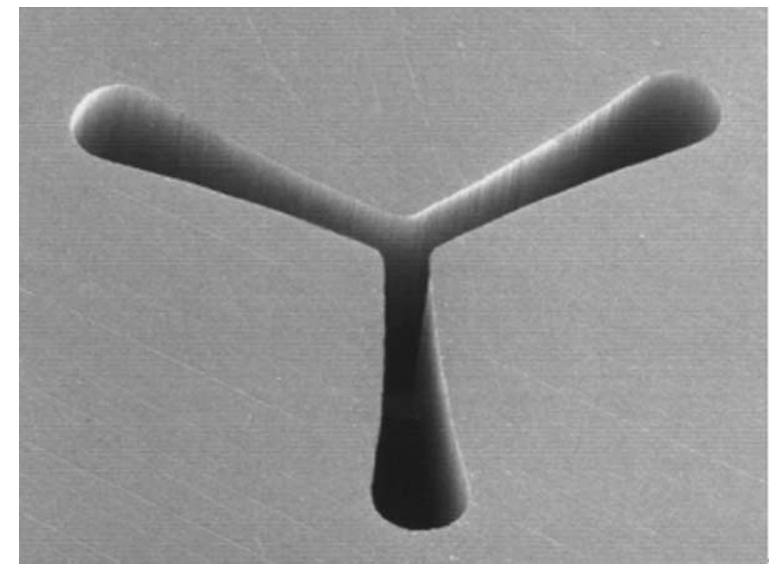

Fig. 1. Spinneret capillary for direct spinning (1 mm deep and $70 \mu \mathrm{m}$ wide).

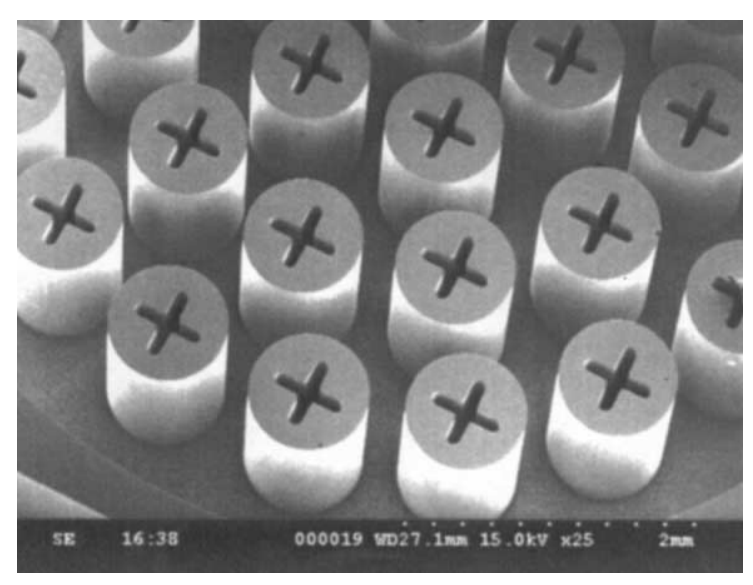

Fig. 2. Spinneret capillary for composite spinning ( $1 \mathrm{~mm}$ in deep and $70 \mu \mathrm{m}$ wide).

any existing manufacturing processes, including EDM.

Fig. 4 is a schematic of the injection molding process. Molten plastic is injected from the spinneret body through guiding holes to form LIGA capillaries. This is followed by the plating process initiated in the steel body of the spinneret. The plated section provides the entire device with the needed mechanical integrity under high pressure spinning conditions. The present plating process produces a very strong bonding at the interface between the steel and plated capillaries. 


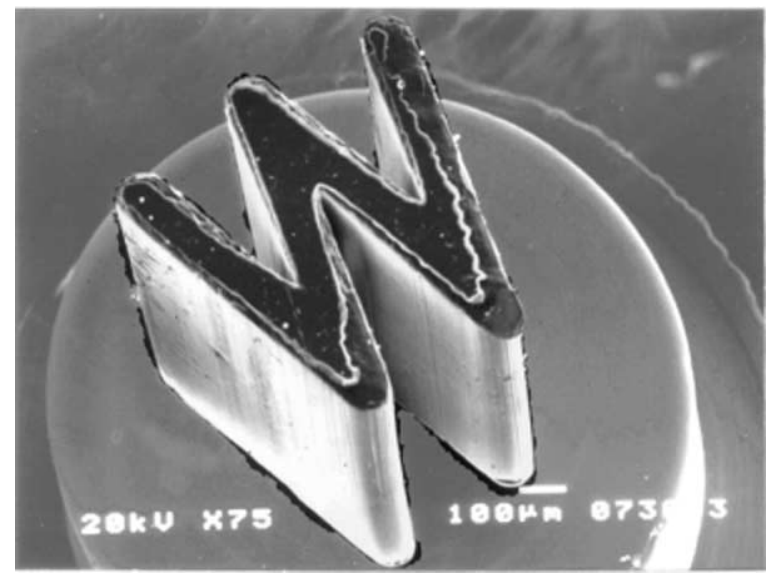

Fig. 3. Complimentary structure of a spinneret capillary manufactured using injection molding $(2 \mathrm{~mm}$ deep and $70 \mu \mathrm{m}$ wide, fabricated at Yunlin University of Science and Technology).

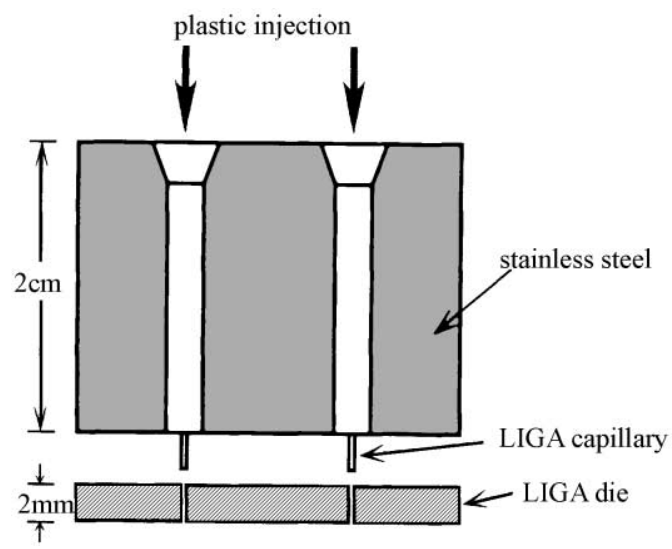

Fig. 4. Duplication process of injection molding. The stainless steel body of the spinneret is, in the meantime, the plating base.

To demonstrate the mass production ability, the injection molding process was performed to duplicate the spinneret capillaries. Fig. 3 shows a close-up picture of a w-shaped capillary made using injection molding. The molding material used in the present study was polypropylene (PP) plus $20 \%$ engineering plastic (ABS). The width of the capillary was $70 \mu \mathrm{m}$, and the depth was $2 \mathrm{~mm}$. Using a single injection process, 72 capillaries were formed, as shown in Fig. 5. The width of each

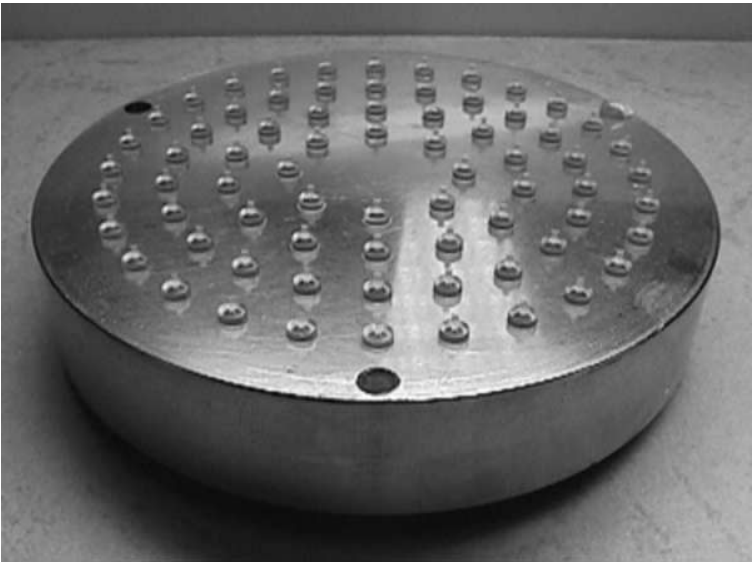

Fig. 5. Duplication of 72 LIGA capillaries by injection molding. The plating process started directly from the stainless steel body.

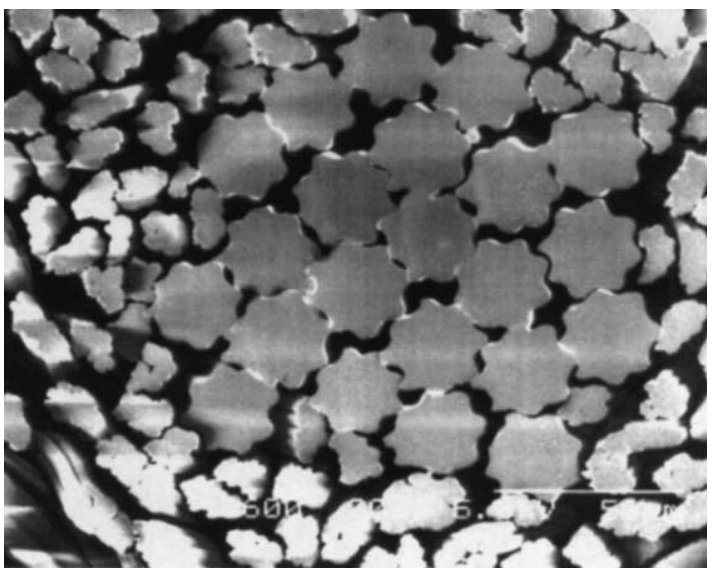

Fig. 6. 22 star-like fibers at the center of the picture are drawn from the LIGA spinneret.

capillary was carefully inspected along the longitudinal direction. The results show that the accuracy of the diameter for each capillary could be controlled to within $\pm 1 \mu \mathrm{m}$.

Fig. 6 shows the fiber drawn from a LIGA spinneret with a cross section of the sign "*” (star). The specific fiber is placed in the middle of photograph with the other fibers together to illustrate the superb shape uniformity. However, the result shows that capillaries of two-millimeter deep are, 
in fact, deeper than the original drawing requirement. Overall, further reduction in fabrication cost is critical for successful commercialization.

\section{Leadframe punch}

Taiwan is one of leading countries in the world for microelectronic IC fabrication and packaging. There are two main methods used to fabricate leadframe, i.e. etching and punching. Although punching is significantly cheaper process than etching, it is very difficult to manufacture highpin-count leadframes using the punching method. One of the main reasons is the increasing precision required for the punch head. The other reason is the increasing cost with the number of pins to be made. The Mechanical Industry Research Laboratory at Industrial Technology Research Institute has recently initiated a project focusing on developing punch production of integrated leadframes based on LIGA technology. The intent here is to use a $\mathrm{NiCo} / \mathrm{SiC}$ alloy composite technology to plate the punch head. The hardness of $\mathrm{NiCo} / \mathrm{SiC}$ composite plating is expected to be higher than that of $\mathrm{Ni} / \mathrm{SiC}$ composite plating.

\section{W-band klystrino and accelerator}

Radio frequency (RF) charged particle acceleration is a special application of millimeter-wave (mm-wave) power source. Theoretically, an accelerator operated with a shorter wavelength can achieve a higher acceleration gradient. This implies that a shorter acceleration distance is necessary to accelerate the particle to a designed energy. Higher acceleration gradients, however, require higher RF power levels, which, in turn, generates more heat. The heating problem is one of the main technical barriers to increase the acceleration gradient for general applications. It is not unreasonable to envision that tabletop synchrotrons will one day play the same roles that lasers play today ranging from deep UV to X-rays. With size specifications in millimeters and precision in micrometers, recent advances in microfabrication technologies have led us to believe that all of these possibilities can be realized in the near future.

Power source and accelerators with planar copper structures designed for the LIGA processes have been discussed in recent years [7]. The critical issue lies on quantifying the relationship between the fabrication depth and the operating frequency. Using the conventional LIGA process, a structure depth of $1 \mathrm{~mm}$ along with an operating frequency of $120 \mathrm{GHz}$ has been considered. For a w-band frequency of $95 \mathrm{GHz}$, which has a wide range of applications due to its superb water transparency, the half wavelength is about $1.5 \mathrm{~mm}$. The UD LIGA process developed at SRRC is one of the best methods to fabricate high-power w-band mmwave devices.

Dark current capture process, in which an accelerating field captures an electron at rest, represents one of the theoretical limitation of an acceleration field. The threshold for capturing an electron raises to a higher level when the operating frequency increases. An acceleration of $1 \mathrm{GV} / \mathrm{m}$ for $95 \mathrm{GHz}$ operation appears to be feasible [8]. While the Stanford Linear Accelerator Center (SLAC) considers building the future linear collider using w-band acceleration, the Berlingroup is designing the standing-wave accelerator for the tabletop synchrotron light source. To avoid the serious heating problem, the tabletop light source will apply lower acceleration gradient. Integration of accelerator parts within a batch

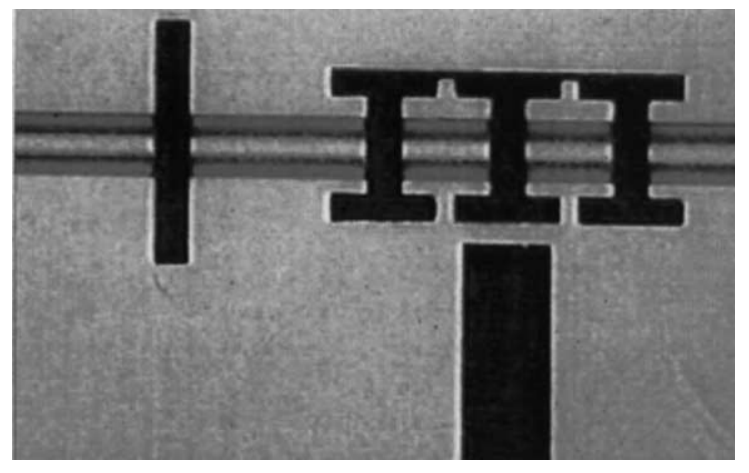

Fig. 7. UD-LIGA fabricated $95 \mathrm{GHz}$ cavities. The photo shows a rectangular penultimate cavity, a three gap output cavity, the output waveguide (iris not cut yet), and the $0.8 \mathrm{~mm}$ diameter beam tunnel. 
process becomes the major concern. The UD LIGA process developed at SRRC has been used to fabricate parts for the Berlin's micro-accelerator. Fig. 7 shows the w-band cavities of the mmwave power source made for such project. Results from the tests performed at SLAC have demonstrated that these cavities are capable of providing a superior $Q$-factor close to its theoretical maximum value.

\section{References}

[1] Y. Cheng, B.Y. Shew, C.Y. Lin, D.H. Wei, Proc. of NSC, Part A, Phys. Sci. Eng. 24 (4) (1999) 537.
[2] Y. Cheng, B.Y. Shew, C.-H. Lin, M.K. Chyu, Sensor Actuators 82 (2000) 205.

[3] Y. Cheng, B.Y. Shew, C.Y. Lin, D.H. Wei, M.K. Chyu, J. Micromech. Microeng. 9 (1999) 58.

[4] Y. Cheng, N.Y. Kuo, C.H. Su, Rev. Sci. Instr. 685 (1997) 2163.

[5] Y. Cheng, C.Y. Lin, D.H. Wei, B. Loechel, G. Gruetzner, IEEE J. MEMS, 81(1999) 18.

[6] B.-Y. Shew, Y. Cheng, C.-H. Lin, W.-P. Ma, G.-J. Hwang, S.-C. Tseng, C.-L. Kuo, G.-L. Chang, D.-S. Lee, Sensors Mater. 11 (6) (1999) 329.

[7] H. Henke, CP398, Advance Accelerator Concepts, S. Chattopadhyay, J. McCullough, P. Dahl (Eds.), AIP press, New York, 1997.

[8] P. Wilson, SLAC-PUB-7449. 